\title{
A molecular dynamics simulation of a water droplet in contact with a platinum surface
}

\author{
Tatsuto Kimura and Shigeo Maruyama \\ Department of Mechanical Engineering, The University of Tokyo, 7-3-1 Hongo, Bunkyo-ku, Tokyo, 113-8656, Japan
}

A water droplet in contact with a platinum surface was simulated by the molecular dynamics method. Water molecules were modeled with the well-known SPC/E model and the platinum surface was represented by three layers of harmonic molecules with the constant temperature heat bath model using the phantom molecules. Two types of water-platinum pair potential functions based on extended Hückel calculations were employed, one was developed by Spohr and Heinzinger in 1988 and the other was proposed by Zhu and Philpott in 1994. In the spreading process of a liquid droplet on a platinum surface, the area of contact region between water and platinum expanded just in proportional to the one-third power of time. This spreading rate was clearly in contrast to the case of Lennard-Jones droplet. Even though the water droplet finally spread to a monolayer film on fcc (111) surface with S-H potential, a stable droplet structure on a monolayer film was realized with Z-P potential. The mechanism of the 'drop on film' structure was explained by the very concentrated monolayer film of water. Comparing three different platinum surface structures, (111), (100) and (110), contact angle varied drastic and it was largest on fcc (100) surface.

\section{Introduction}

Molecular level understanding of dynamics of liquid and solid interface is important for phase change heat transfer such as evaporation and condensation. The authors have presented studies on equilibrium liquid droplet (Maruyama et al., 1998), vapor bubble nucleation (Maruyama and Kimura, 2000), and liquid droplet nucleation (Kimura and Maruyama and, 2002) for the simple Lennard-Jones fluid system. By arbitrary changing the potential parameter between fluid and solid molecules, considerable information is obtained about the wetting of surface. In practice, water is the most important molecule in engineering and in theoretical framework. Here, a molecular dynamics simulation of a water droplet on a platinum surface is performed. The spreading phenomena and final equilibrium structure of water on the surface were compared for two different water-platinum intermolecular potentials and different platinum surface lattice structures.

\section{Simulation method}

The calculation system is shown in Figure 1. Platinum surface was located at the bottom and mirror boundary condition at the top and periodic boundary conditions in 4 sides were employed. Water molecule was modeled with the well-known SPC/E model (Berendsen et al., 1987) and the platinum surface was represented by three layers of harmonic molecules. The phantom molecules (Blömer and Beylich, 1999) were used beneath the layers

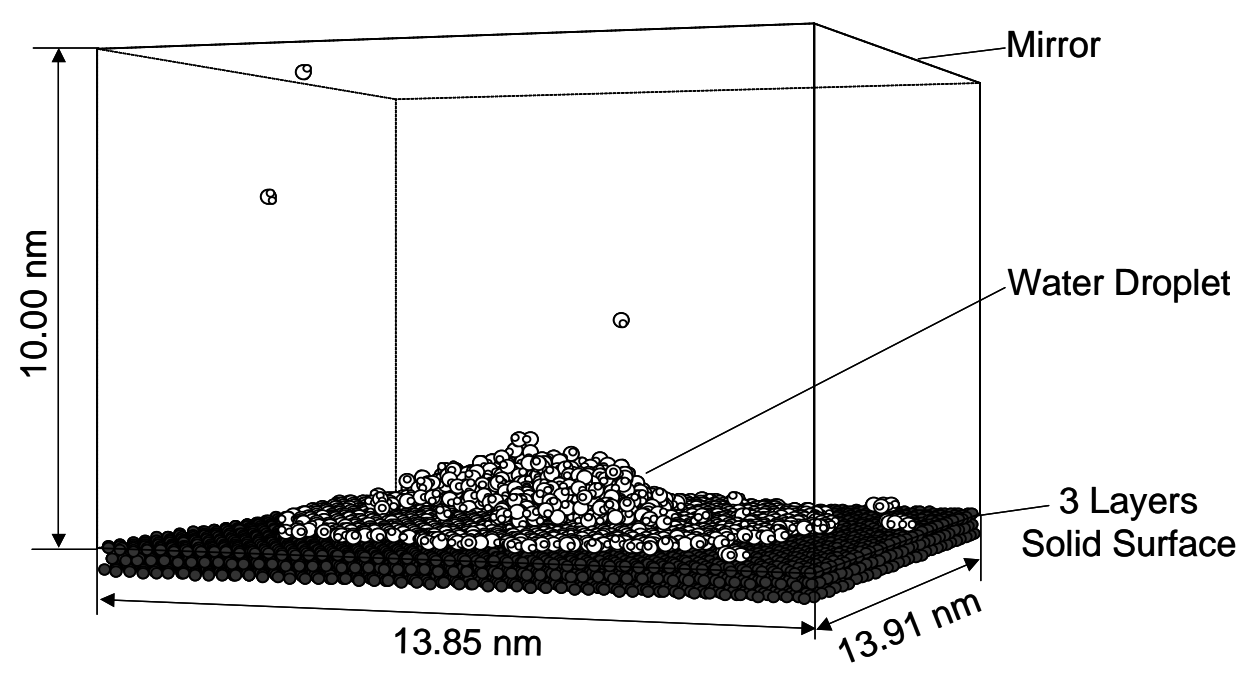

Fig. 1 System configuration (fcc (111) surface). A snapshot of equilibrium structure at $350 \mathrm{~K}$ with Z-P potential. 
of platinum surface in order to mimic the constant temperature heat bath. Harmonic potential parameters for platinum atoms were set as $k=46.8 \mathrm{~N} / \mathrm{m}$ (spring constant) and $r_{0}=0.277 \mathrm{~nm}$ (nearest neighbor distance).

Two different potential functions between water and platinum surface were employed. Both potential models were derived from the extended Hückel calculations between a water molecule and a platinum cluster (Holloway and Bennemann, 1980). One is the model developed by Spohr and Heinzinger in 1988 (S-H potential). The potential function is described as follows.

$$
\begin{aligned}
& \phi_{\mathrm{H}_{2} \mathrm{O}-\mathrm{Pt}}=\phi_{\mathrm{O}-\mathrm{Pt}}\left(r_{\mathrm{OPt}}, \rho_{\mathrm{OPt}}\right)+\phi_{\mathrm{HPt}}\left(r_{\mathrm{H}_{1} \mathrm{Pt}}\right)+\phi_{\mathrm{H}-\mathrm{Pt}}\left(r_{\mathrm{H}_{2} \mathrm{Pt}}\right) \\
& \phi_{\mathrm{O}-\mathrm{Pt}}=\left[a_{1} \exp \left(-b_{1} r\right)-a_{2} \exp \left(-b_{2} r\right)\right] f(\rho)+a_{3} \exp \left(-b_{3} r\right)[1-f(\rho)] \\
& \phi_{\mathrm{H}-\mathrm{Pt}}=a_{4} \exp \left(-b_{4} r\right) \\
& f(\rho)=\exp \left(-c \rho^{2}\right) \\
& a_{1}=1.8942 \times 10^{-16} \mathrm{~J}, \quad b_{1}=11.004 \mathrm{~nm}^{-1} \\
& a_{2}=1.8863 \times 10^{-16} \mathrm{~J}, \quad b_{2}=10.966 \mathrm{~nm}^{-1} \\
& a_{3}=10^{-13} \mathrm{~J}, \quad b_{3}=53.568 \mathrm{~nm}^{-1} \\
& a_{4}=1.742 \times 10^{-19} \mathrm{~J}, \quad b_{4}=12.777 \mathrm{~nm}^{-1} \\
& c=11.004 \mathrm{~nm}^{-1}
\end{aligned}
$$

Here, $r$ is inter-atomic distance, $\rho$ is the length of the projection of the distance vector onto the surface plane. This potential has the minimum value when a water molecule sits on top of a platinum atom with the dipole moment directing upward. Figure 2 shows configurations of small cluster of water on the surface. Because of the hydrogen bonding of water molecules, each water molecules has different orientation from the single water case.

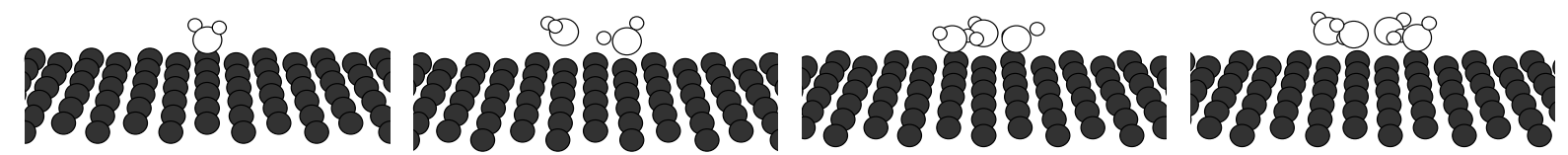

(a) One molecule

(b) Two molecules

(c) Three molecules

(d) Four molecules

Fig.2 Small water clusters on fcc (111) platinum surface at $0 \mathrm{~K}$ (S-H potential).

The other potential model between water and platinum surface was developed by Zhu and Philpott in 1994 (Z-P potential). The potential function consists of water molecule-conduction electron potential, anisotropic short-range potential and isotropic short-range $\mathrm{r}^{-\mathrm{n}}$ potential as follows.

$$
\begin{aligned}
& \phi_{\mathrm{H}_{2} \mathrm{O}-\mathrm{surf}}=\phi_{\mathrm{H} 2 \mathrm{O}-\mathrm{cond}}+\phi_{\mathrm{an}}\left(\mathrm{O} ; \mathbf{r}_{\mathrm{O}}\right)+\phi_{\mathrm{isr}}\left(\mathrm{O} ; \mathbf{r}_{\mathrm{O}}\right)+\sum_{H}\left[\phi_{\mathrm{an}}\left(\mathrm{H} ; \mathbf{r}_{\mathrm{H}}\right)+\phi_{\mathrm{isr}}\left(\mathrm{H} ; \mathbf{r}_{\mathrm{H}}\right)\right] \\
& \phi_{\mathrm{H} 2 \mathrm{O}-\mathrm{cond}}=\sum_{l, k} \frac{q_{l} q_{k}}{2 r_{l k}} \\
& \phi_{\mathrm{an}}\left(p ; \mathbf{r}_{p}\right)=4 \varepsilon_{p-\mathrm{Pt}} \sum_{j}\left[\left(\frac{\sigma_{p-\mathrm{Pt}}^{2}}{\left(\alpha \rho_{p j}\right)^{2}+z_{p j}^{2}}\right)^{6}-\left(\frac{\sigma_{p-\mathrm{Pt}}^{2}}{\left(\rho_{p j} / \alpha\right)^{2}+z_{p j}{ }^{2}}\right)^{3}\right] \\
& \phi_{\mathrm{isr}}\left(p ; \mathbf{r}_{p}\right)=-4 \varepsilon_{p-\mathrm{Pt}} \sum_{j} \frac{c_{p-\mathrm{Pt}} \sigma_{p-\mathrm{Pt}}^{10}}{r_{p j}^{10}} \\
& \alpha=0.8 \\
& \sigma_{\mathrm{O}-\mathrm{Pt}}=0.270 \mathrm{~nm}, \varepsilon_{\mathrm{O}-\mathrm{Pt}}=6.44 \times 10^{-21} \mathrm{~J}, c_{\mathrm{O}-\mathrm{Pt}}=1.28 \\
& \sigma_{\mathrm{H}-\mathrm{Pt}}=0.255 \mathrm{~nm}, \varepsilon_{\mathrm{H}-\mathrm{Pt}}=3.91 \times 10^{-21} \mathrm{~J}, c_{\mathrm{H}-\mathrm{Pt}}=1.2
\end{aligned}
$$


Equation (6) represents Coulomb potential between point charge of water molecule and its image charge located at symmetrical position below the image plane. This potential also has the minimum value when a water molecule sits on top of a platinum atom, but the binding energy is stronger than S-H potential.

The well-known Ewald method is usually used for the long-range correction of Coulomb potential (Allen and Tildesley, 1987). However, we used the cut-off method (Wolf et al., 1999) in order to increase the computational efficiency for this heterogeneous system. The large cut-off length of $25 \AA$ between water and water molecules and $15 \AA$ between water and platinum atoms were employed. The leapfrog method is used for the numerical integration of the equation of motion with the time step of $0.5 \mathrm{fs}$.

As the initial condition, an ice crystal with 856 or 2048 molecules with the density of bulk water at $350 \mathrm{~K}$ was placed on the center of the platinum surface. For the initial $100 \mathrm{ps}$, simple velocity scaling was used for the temperature control. After this initial rough control, only the phantom heat bath was employed as the temperature control at $350 \mathrm{~K}$.

\section{Spreading of a water droplet on a platinum surface}

Figure 3 shows snapshots of the water droplet with 2048 molecules on fcc (111) platinum surface employing Spohr-Heinzinger water-platinum potential. During the velocity scaling temperature control in $100 \mathrm{ps}$, the liquid vapor interface was already in spherical cap shape. Then, the gradual spreading of the water droplet was observed. Figure 4 shows the change in total dipole moment of the system. The increase of the total dipole moment is the good measure of the average orientation of water molecules. Since the contribution to the total dipole moment from a relatively free water molecule is large, the increase in the dipole moment shows the spreading. The smallest system with 856 water molecules reached the equilibrium at about 1000 ps with the almost constant total dipole moment. And for the larger 2048 system, the equilibrium was almost obtained at 2000 ps.

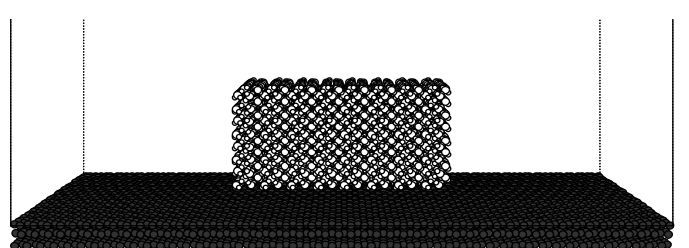

(a) $0 \mathrm{ps}$

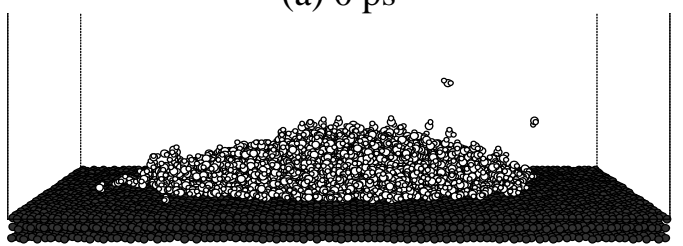

(c) $200 \mathrm{ps}$

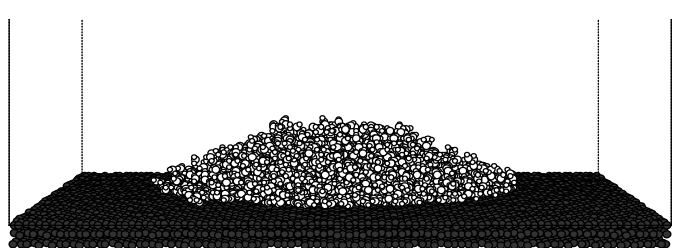

(b) $100 \mathrm{ps}$

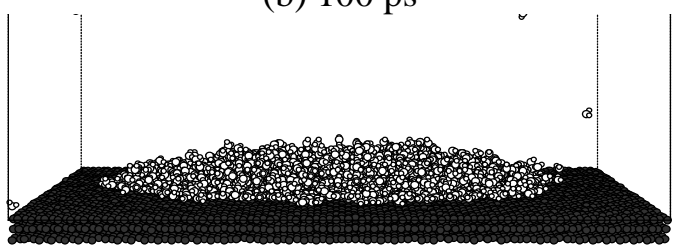

(d) $300 \mathrm{ps}$

Fig. 3 Snapshots of water droplet on fcc (111) surface (S-H potential).

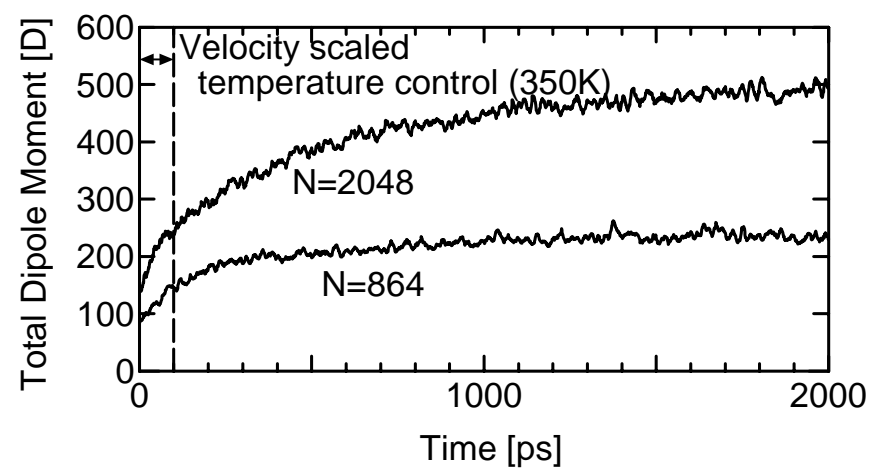

Fig. 4 Variations of total dipole moment of the system (fcc (111), S-H potential). 
The spreading of the water droplet is measured in Figure 5 as the area of water-platinum interface. Except for the initial 100 ps when the velocity-scaling temperature control was done, this change of the interface area was proportional to the one-third power of time. Here, the deviation from the straight line is observed at the end of simulation, when the equilibrium condition is approached. The broken lines are fit lines with $A \propto t^{1 / 3}$, or $R \propto t^{1 / 6}$ with the nominal radius of the first layer. Inspired by the direct ellipsometric measurements of monolayer and terraced liquid film (Heslot et al., 1990), spreading of liquid on completely wet surface has been studied extensively in this decade. Even though the experimentally assigned spreading rate was $A \propto t$, initial molecular dynamics simulations using Lennard-Jones potential resulted in contradictory relations, $A \propto \log (t)$ (Young et al., 1992) or $A \propto t^{2}$ (Nieminen et al., 1992), depending on the volatility or the surface conditions. More recent large-scale molecular dynamics simulations could predict the correct spreading rate; $A \propto t$ and extensive efforts of the modeling were being performed (Voué and Coninck, 2000). The much slower spreading rate of water on the platinum surface compared with the case of simple molecules is probably because of the large friction of the contact line movement due to the commensuration of water layer to the platinum crystal.

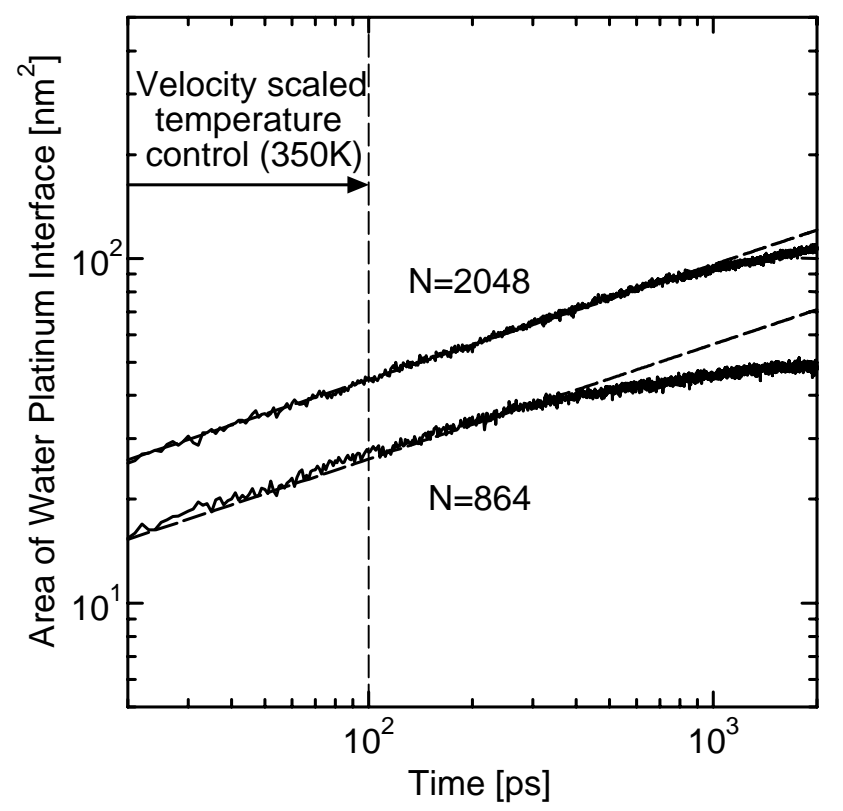

Fig. 5 Expansion of water-platinum contact area (fcc (111), Spohr-Heinzinger potential).

\section{Structure of water droplet on platinum surface}

Figure 6 shows the two-dimensional density distributions of the water droplets for 2048 water molecules system averaged from 1900 ps to 2000 ps. In the case of S-H potential, the droplet finally spread to almost monolayer and only a few molecules stayed on the film. On the other hand, in the case of Z-P potential, a liquid droplet with a contact angle on the monolayer was observed. This is the first realization of stable droplet structure on the precursor film with molecular dynamics level calculation. Even though existence of the precursor film is anticipated, the clear physical understanding of the three phase contact line has not been obtained. The simple relation obtained for Lennard-Jones fluid was that the interface potential strength was proportional to the cosine of contact angle (Maruyama et al., 1998). This could be really imagined by the analogy to the Young's equation. However, the stronger interaction in Z-P potential now resulted the less wetting structure compared with S-H potential. The reason for this apparently controversial result can be explained as follows. The monolayer was very dense and there was space between the monolayer and the droplet. The dense monolayer repulses the other molecules, so that the effective potential between a platinum surface and the droplet on the monolayer is weakened. Hence, the layer concentration of the monolayer leads to the larger contact angle (less wettable). 


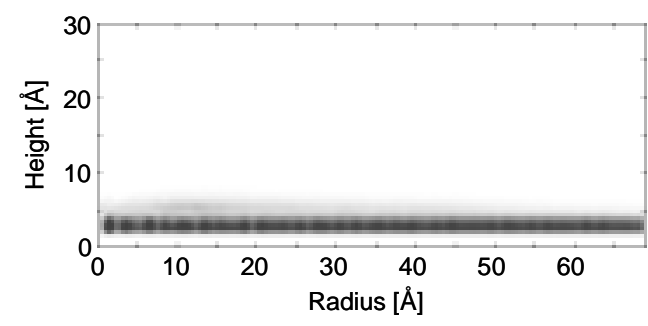

(a) Spohr-Heinzinger potential

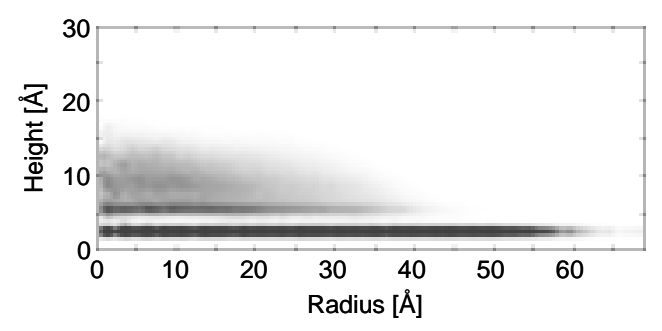

(b) Zhu-Philpott potential

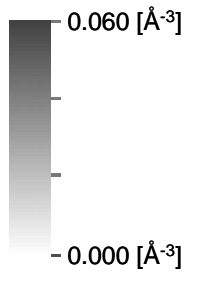

Fig. 6 Two-dimensional density profiles of water droplet on fcc (111) platinum surface.

Figure 7 and Figure 8 show snapshots of the water droplet and its first layer on different surface lattice structures employing S-H potential and Z-P potentials respectively. The platinum surface atom density is largest for (111), medium for (100) and smallest for (110). For (100) and (110) water molecules almost completely cover the platinum atoms. Hence, the water molecular density of monolayer is largest for (100) surface, and the contact angle is the largest. In the case of (110), the platinum atom density is very low and the contact angle is the smallest. These trends were valid for both S-H (weaker) and Z-P (stronger) potentials. Hence, the explanation based on the monolayer density is completely valid.

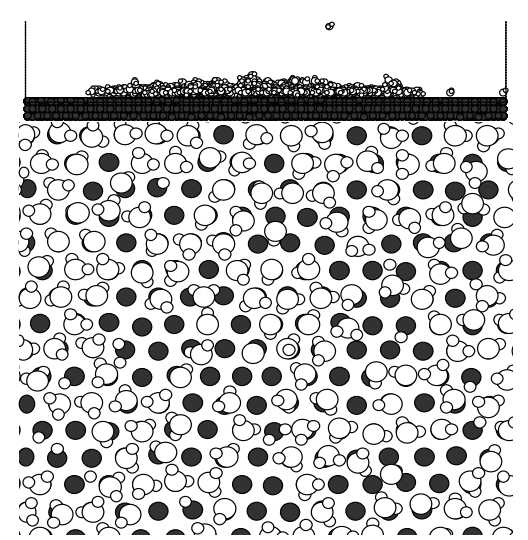

(a) fcc (111)

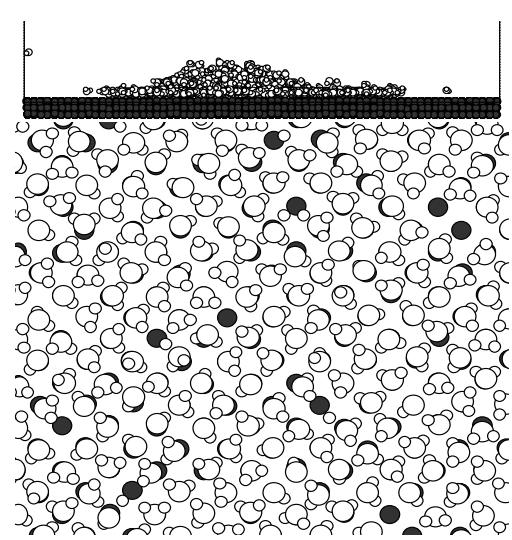

(b) fcc (100)

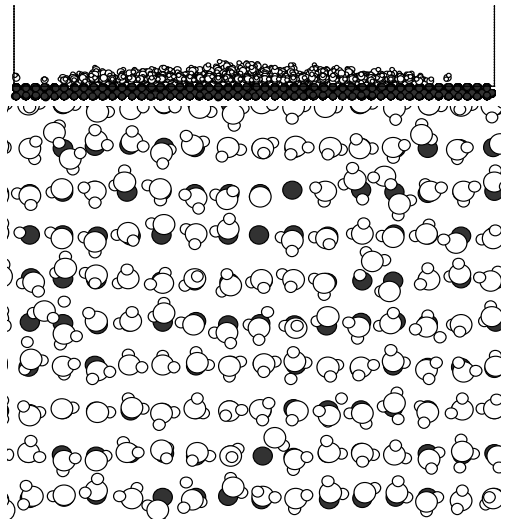

(c) fcc (110)

Fig.7 Snapshots of water droplet and its first layer (Spohr-Heinzinger potential).

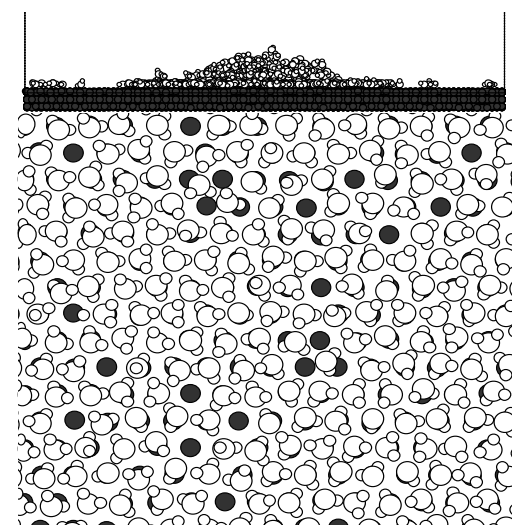

(a) fcc (111)

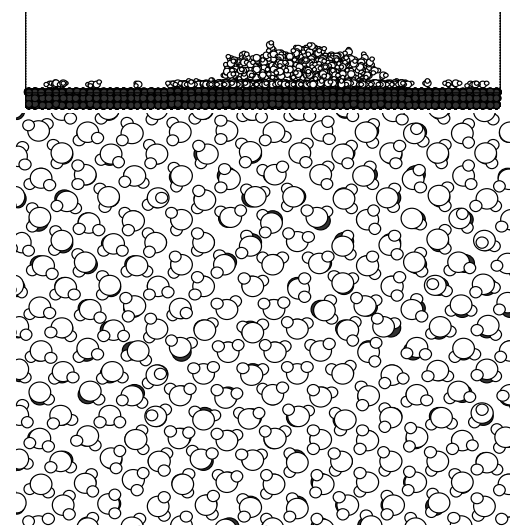

(b) fcc (100)

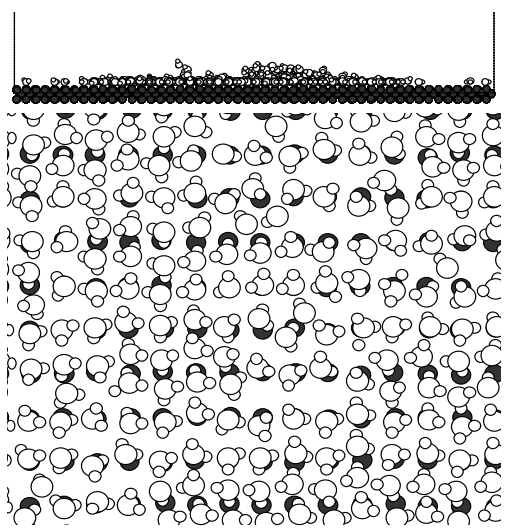

(c) fcc (110)

Fig.8 Snapshots of water droplet and the first layer (Zhu-Philpott potential). 


\section{Conclusions}

A molecular dynamics simulation of a water droplet on a platinum surface was performed. In the spreading process of water droplet, the area of contact region between water and platinum expanded just in proportional to the one-third power of time. This speed is slower than the case of Lennard-Jones liquid droplet. The final equilibrium shape of a water droplet was compared between two different water-platinum potentials and three different platinum surface lattice structures. With stronger water-platinum interaction, the contact angle became larger because of the repulsive effect of the dense monolayer. On fcc (100) platinum surface, because of high coverage of water molecules on platinum atoms, the contact angle was largest.

\section{References}

M. P. Allen and D. J. Tildesley, Computer Simulation of Liquids, Oxford University Press, New York, 1987.

H. J. C. Berendsen, J. R. Grigera and T. P. Straatsma, The Missing Term in Effective Pair Potentials, J. Phys. Chem., vol. 91, no. 24, pp.6269-6271, 1987.

J. Blömer and A. E. Beylich, Molecular Dynamics Simulation of Energy Accommodation of Internal and Translational Degrees of Freedom at Gas-Surface Interfaces, Surf. Sci., vol. 423, pp. 127-133, 1999.

F. Heslot, A. M. Cazabat, P. Levinson and N. Fraysse, Experiments on Wetting on the Scale of Nanometers: Influence of the Surface Energy, Phys. Rev. Lett., vol. 65, no. 5, pp. 599-602, 1990.

S. Holloway and K. H. Bennemann, Study of Water Adsorption on Metal Surfaces, Surf. Sci., vol. 101, pp. 327-333, 1980.

T. Kimura and S. Maruyama, A Molecular Dynamics Simulation of Heterogeneous Nucleation of a Liquid Droplet on Solid Surface, Microscale Thermophys. Eng., vol. 6, no. 1, in print, 2002.

S. Maruyama, T. Kurashige, S. Matsumoto, Y. Yamaguchi and T. Kimura, Liquid Droplet in Contact with a Solid Surface, Microscale Thermophys. Eng., vol. 2, no. 1, pp.49-62, 1998.

S. Maruyama and T. Kimura, A Molecular Dynamics Simulation of a Bubble Nucleation on Solid Surface, Int. J. Heat \& Technology, vol. 8, no. 1, pp.69-74, 2000.

J. A. Nieminen, D. B. Abraham, M. Karttunen and K. Kaski, Molecular Dynamics of a Microscopic Droplet on Solid Surface, Phys. Rev. Lett., vol. 69, no. 1, pp. 124-127, 1992.

E. Spohr and K. Heinzinger, A Molecular Dynamics Study on the Water/Metal Interfacial Potential, Ber. Bunsenges. Phys. Chem., vol. 92, pp. 1358-1363, 1988.

M. Voué and J. De Coninck, Spreading and Wetting at the Microscopic Scale: Recent Developments and Perspectives, Acta Mater., vol. 48, pp. 4405-4417, 2000.

D. Wolf, P. Keblinski, S. R. Phillpot and J. Eggebrecht, Exact Method for the Simulation of Coulombic System by Spherically Truncated, Pairwise ${ }^{-1}$ Summation, J. Chem. Phys., vol. 110, no. 17, pp. 8254-8282, 1999.

J. Young, J. Koplik and J. R. Banavar, Terraced Spreading of Simple Liquids on Solid Surfaces, Phys. Rev. A, vol. 46, no. 12, pp. 7738-7749, 1992.

S.-B. Zhu and M. R. Philpott, Interaction of Water with Metal Surfaces, J. Chem. Phys., vol. 100, no. 9, pp. 6961-6968, 1994. 